\title{
APPLICATION OF MOLECULAR IMPRINTED POLYMERS FOR SELECTIVE SOLID PHASE EXTRACTION OF BISPHENOL A
}

\author{
ZASTOSOWANIE POLIMERÓW Z NADRUKIEM CZĄSTECZKOWYM \\ DO SELEKTYWNEJ EKSTRAKCJI DO FAZY STAŁEJ BISFENOLU A
}

\begin{abstract}
Selective molecularly imprinted polymers (MIPs) with bisphenol A as template were synthesized using the non-covalent imprinting approach. MIPs were prepared using thermally initiated polymerization with $1,1^{\prime}$-azobis(cyclohexanecarbonitryle) (ACHN) as initiator and ethylene glycol dimethacrylate (EDMA) as a cross-linking agent. The tested functional monomers included methacrylic acid, acrylamide, and 4-vinylpyridine. The selectivity of the BPA-MIP for the solid phase extraction of bisphenol A was tested in samples containing other related alkylphenols. The polymers prepared in acetonitrile using methacrylic acid or acrylamide as monomer showed the highest selectivity towards target analyte (the selectivity ratio 8:1, respectively for MIP and NIP). The proposed procedure has been proven to be an effective for selective extraction of bisphenol A in aqueous samples (recoveries over 85\%) enabling detection and quantification limits of 25 and $70 \mu \mathrm{g} / \mathrm{dm}^{3}$, respectively based on $10 \mathrm{~cm}^{3}$ of sample volume, with relative standard deviations (RSD) lower than $6 \%$. The obtained molecularly imprinted material showed interesting properties for selective extraction and preconcentration of studied analyte from large volumes of aqueous samples without any problems of cartridge clogging.
\end{abstract}

Keywords: bisphenol A, molecularly imprinted polymer, 1,1'-azobis(cyclohexanecarbonitryle), water analysis, solid-phase extraction

\section{Introduction}

In the last years, many papers describing the occurrence of chemicals (xenobiotics) responsible for disrupting the proper function of living animals (especially water organisms) have been published [1-14]. These studies also apply to the determination of bisphenol A (BPA, 2,2-bis(4-hydroxylphenyl)propane) that use has been a point of controversy for the recent years [15-19].

The various tests in rodents proven that BPA competed with $(3 \mathrm{H})$ estradiol for binding to estrogen receptors (ER) from rat uterus [17]. It has been demonstrated, that BPA leads to

\footnotetext{
${ }^{1}$ Faculty of Chemistry, Opole University, pl. M. Kopernika 11a, 45-040 Opole, Poland, phone +48 774527116 , fax +48 7745271 15, email: Piotr.Wieczorek@uni.opole.pl

${ }^{2}$ Institute of Heavy Organic Synthesis "Blachownia", ul. Energetyków 9, 47-225 Kędzierzyn-Koźle, Poland, phone +48 7748734 92, fax +48 7748730 60, email: moscipan.m@icso.com.pl

"Corresponding author: Anna.Poliwoda@uni.opole.pl
} 
sterility among males, reduces in milk production among females and fosters to the development of cancer $[15,16]$. Also, the estrogenic activity of saliva samples collected from patients with a fissure sealant was strongly related to the BPA content $[20,21]$. The source of bisphenol A is polycarbonate plastics, phenolic and epoxy resins (used for lining food and beverage cans) as well as dental sealants where this chemical was used as an important intermediate (e.g. as an antioxidant or polymerization inhibitor). In this case, incomplete conversion of monomers during the polymerization process can leach out of the bisphenol A, from such materials into the environment, especially during heating of the cans or contact with basic or acidic substances [22, 23]. It seems that bisphenol A can easily get into the environment, widely exist there and finally migrate into the human body to produce adverse effects on health [24]. Therefore the monitoring of these endocrine disrupting chemicals should be of immense importance.

Reported concentration of bisphenol A in environmental samples is very low, and the application of specific preconcentration and sample clean-up steps, before the use of highly sensitive analytical techniques are mandatory [25-28]. For this purposes, the various types of extraction methods such as solid phase extraction (SPE) [20, 27, 29-33], liquid-liquid extraction (LLE), accelerated solvent extraction (ASE) [34] as well as membrane extraction (ME) $[35,36]$ have been widely applied. In many cases, the techniques such as LLE, ASE, and $\mathrm{ME}$ made possible an efficient isolation of studied analyte, whereas, for preconcentration step, a using of subsequent solid phase extraction cartridges was necessary to be performed additionally. The commercially available extraction cartridges possess some drawbacks like the low selectivity and column clogging when large amounts of samples are loaded. The application of molecularly imprinted polymers (MIPs), as sorbents in solid phase extraction approach, might overcome this limitation [37-41]. These polymers have many advantages, such as selectivity, the simplicity of implementation and low-cost materials. Additionally, MIPs are reusable and have high stability and resistance to acids, organic solvents, and high temperatures. It has been reported that BPA-imprinted MIPs prepared by various imprinting techniques have been successfully used as SPE sorbents to different samples for low BPA concentration [37, 39, 42-50]. In the most cases, the BPA-imprinted polymers exhibited higher binding affinities than non-imprinted polymers toward BPA extraction. However, the selectivity studies of BPA extraction from the mixtures of several related structural analogs with an application of synthesized BPA-MIPs and various solvents during washing step were not always satisfied enough. In fact, the increase of the volume of washing solvent during MISPE procedure decreased the BPA enrichment effect whereas the obtained recoveries values for its analogs did not significantly change [47, 49]. Often, a compromise solution for washing step was required to be employed to obtain a specific selectivity.

This study demonstrates the synthesis of BPA - molecularly imprinted polymers using non-covalent polymerization technique and their use to selectively extract and concentrate bisphenol A molecules from water samples containing other phenolic structural analogs. Here, we have examined the selectivity and binding recognition properties of obtained BPA-MIP depending on the application of different functional monomers (methacrylic acid (MAA), acrylamide (AA) and 4-vinylpiridyne (4-VP)) as well as porogenic solvents. Furthermore, this work also focused on the evaluation of specific molecularly imprinted solid phase extraction (MISPE) for selective and continuous isolation and preconcentration of BPA in the presence of other structurally related compounds (other alkylphenols). The MISPE extracts analyzed using HPLC with diode array detection (PDA). The imprinted 
sorbent with the best molecular recognition abilities was successfully applied to the analysis of bisphenol $\mathrm{A}$ in real river water samples showing the applicability of proposed analytical procedure.

\section{Experimental}

\section{Materials}

Methacrylic acid (MAA), 4-vinylpirydine (4-VP), acrylamide (AA), ethylene glycol dimethacrylate (EDMA), bisphenol A (BPA), nonylphenol (NP), 4-tert-octylphenol (t-OP) and 2-phenylphenol (2-PP) were purchased from Sigma-Aldrich (Poznan, Poland). The thermally decomposing initiator, a 1,1'-azobis (cyclohexanecarbonitryle) (ACHN, Sigma-Aldrich, purity > 98\%) was used as received and kept in a freezer. Anhydrous solvents for polymers synthesis: chloroform $\left(\mathrm{CHCl}_{3}\right)$, toluene $\left(\mathrm{C}_{6} \mathrm{H}_{5} \mathrm{CH}_{3}\right)$, acetonitrile $\left(\mathrm{CH}_{3} \mathrm{CN}\right)$ and dichloromethane $\left(\mathrm{CH}_{2} \mathrm{CL}_{2}\right)$ as well as all other solvents were of analytical grade and were purchased from POCH (Gliwice, Poland). The monomers: methacrylic acid (MAA) and 4-vinylpiridine (4VP) were purified by vacuum distillation to remove the polymerization inhibitor. EDMA was purified with extraction method by using $10 \%$ solution of sodium hydroxide, then saturated solution of sodium chloride and dried over anhydrous magnesium sulfate. HPLC grade acetonitrile was from J.T. Baker (Mallinckrodt Baker, Deventer, The Netherlands). Water was purified in our laboratory using a Milli-Q system from Millipore (Bedford, MA, USA). Stock solutions of each analyte were prepared at $1 \mathrm{mg} / \mathrm{cm}^{3}$ in methanol and stored at $4^{\circ} \mathrm{C}$ in the dark. Working solutions were daily prepared by the appropriate dilution of the stock solutions.

\section{Synthesis of bisphenol A-imprinted polymers}

Polymers imprinted (MIPs) with bisphenol A were prepared by bulk polymerization, using the non-covalent approach with the application of three various types of functional monomers (methacrylic acid, acrylamide or 4-vinylpirydyne) and ethylene glycol dimethacrylate (EDMA) as a crosslinking monomer. Chloroform, toluene $\left(\mathrm{C}_{6} \mathrm{H}_{5} \mathrm{CH}_{3}\right)$, acetonitrile $\left(\mathrm{CH}_{3} \mathrm{CN}\right)$ and dichloromethane $\left(\mathrm{CH}_{2} \mathrm{CL}_{2}\right)$ were used as a porogen. $0.25 \mathrm{mmol}$ of BPA, 4 mmol of appropriate functional monomer, 20 mmol of EDMA and $1 \%$ of ACHN as radical initiator were dissolved in $12 \mathrm{~cm}^{3}$ of porogen in a glass tube. The test tube with the polymerization mixture was purged with nitrogen for 5 minutes. The polymerization was thermally initiated at $60^{\circ} \mathrm{C}$, and the reaction was carried out for 24 hours. The obtained solid polymers were crushed and sieved (using a 250 mesh sieve). Template removal was performed by solid phase extraction using methanol as an elution solvent, resulting in 3 solvent cycles $\left(3 \cdot 10 \mathrm{~cm}^{3}\right)$. To estimate the effectiveness of BPA removal, $1 \mathrm{~cm}^{3}$ aliquots of the extracts were taken after each SPE procedure and were analyzed by HPLC-PDA system. Non imprinted polymers (NIP) were prepared under identical conditions without the addition of template molecule - BPA. The details about synthesized polymers, their compositions were shown in Table 1.

\section{MISPE condition}

Solid phase extraction was performed using 12-position manifold of Baker with a vacuum pump (KNF Neuberger Laboport, Trenton, New Jersey). $100 \mathrm{mg}$ of molecularly imprinted polymers were packed with $\mathrm{MeOH}$ into PTFE empty SPE cartridge ( $1 \mathrm{~cm} \mathrm{I.D.,}$ $6 \mathrm{~cm}$ long, $3 \mathrm{~cm}^{3}$ volume, Supelco, Bellefonte, USA), protected from both side with Teflon 
frits also from Supelco. Before samples were processed, conditioning of the polymer with $1 \mathrm{~cm}^{3}$ of methanol and $1 \mathrm{~cm}^{3}$ of Milli-Q water was performed. Then $10 \mathrm{~cm}^{3}$ of a sample containing studied analyte(s) was loaded. Elution solvent was $1.5 \mathrm{~cm}^{3}$ of mixture consisted of methanol, acetic acid, and water in ratio $95: 2.5: 2.5_{\mathrm{v} / \mathrm{v} / \mathrm{v}}$, respectively. The polymer was regenerated by passing $5 \mathrm{~cm}^{3}$ of methanol and $5 \mathrm{~cm}^{3}$ of water.

Table 1

The type and chemical composition of synthesized molecularly imprinted polymers

\begin{tabular}{|c|c|c|c|c|}
\hline Polymer & Porogen & Templete - BPA [mmol] & Crosslinker [mmol] & Functional monomer [mmol] \\
\hline MIP 1 & $\mathrm{CH}_{3} \mathrm{CN}$ & 0.25 & 20 & MAA [4 mmol] \\
\hline MIP 2 & $\mathrm{CHCl}_{3}$ & 0.25 & 20 & MAA [4 mmol] \\
\hline MIP 3 & $\mathrm{C}_{6} \mathrm{H}_{5} \mathrm{CH}_{3}$ & 0.25 & 20 & MAA [4 mmol] \\
\hline MIP 4 & $\mathrm{CH}_{2} \mathrm{Cl}_{2}$ & 0.25 & 20 & MAA [4 mmol] \\
\hline MIP 5 & $\mathrm{CH}_{3} \mathrm{CN}$ & 0.25 & 20 & AA [4 mmol] \\
\hline MIP 6 & $\mathrm{CH}_{3} \mathrm{CN}$ & 0.25 & 20 & AA [4 mmol] \\
\hline MIP 7 & $\mathrm{CHCl}_{3}$ & 0.25 & 20 & AA [4 mmol] \\
\hline MIP 8 & $\mathrm{C}_{6} \mathrm{H}_{5} \mathrm{CH}_{3}$ & 0.25 & 20 & MAA [4 mmol] \\
\hline MIP 9 & $\mathrm{CH}_{3} \mathrm{CN}$ & 0.25 & 10 & MAA [2 mmol] \\
\hline MIP 10 & $\mathrm{CH}_{3} \mathrm{CN}$ & 0.25 & 30 & MAA [6 mmol] \\
\hline MIP 11 & $\mathrm{CH}_{3} \mathrm{CN}$ & 0.25 & 20 & MAA [4 mmol] \\
\hline MIP 12 & $\mathrm{CH}_{3} \mathrm{CN}$ & 0.25 & 20 & MAA [4 mmol] \\
\hline MIP 13 & $\mathrm{CH}_{2} \mathrm{Cl}$ & 0.25 & 10 & MAA [4 mmol] \\
\hline MIP 14 & $\mathrm{CH}_{3} \mathrm{CN}$ & 0.50 & 20 & MAA [4 mmol] \\
\hline MIP 15 & $\mathrm{CH}_{2} \mathrm{Cl}$ & 0.50 & 20 & \\
\hline MIP 16 & $\mathrm{CH}_{3} \mathrm{CN}$ & 1.0 & \multicolumn{3}{l}{} \\
\hline
\end{tabular}

\section{HPLC analysis}

Chromatographic analysis was carried out with Dionex UltiMate 3000 HPLC system (Sunnyvale, CA, USA) equipped with a quaternary pump, an on-line degasser, an autosampler, an automatic injector, a column thermostat and diode array detector (PDA). Data acquisition was controlled by Chromoleon series software from Dionex. Phenolic compounds were analyzed on Microsorb-MV, C18 (150 mm x $4.6 \mathrm{~mm}$ I.D., $5 \mu \mathrm{m}, 100 \AA)$ HPLC column protected by a MetaGuard column (4.6 mm MonoChrom, $5 \mu \mathrm{m}, \mathrm{C} 18$ ) both from Varian (Palo Alto, USA). Gradient elution of acetonitrile (A) and water (B) as elution solvents delivered at a constant flow of $1 \mathrm{~cm}^{3} / \mathrm{min}$ was applied. The elution profile was programmed as follows: $\mathrm{t}=0 \min 50 \mathrm{~A}: 50 \mathrm{~B} ; \mathrm{t}=5 \min 65 \mathrm{~A}: 35 \mathrm{~B} ; \mathrm{t}=15 \min 95 \mathrm{~A}: 5 \mathrm{~B}$; $\mathrm{t}=30 \min 95 \mathrm{~A}: 5 \mathrm{~B} ; \mathrm{t}=35 \min 65 \mathrm{~A}: 35 \mathrm{~B} ; \mathrm{t}=40 \min 50 \mathrm{~A}: 50 \mathrm{~B}$. For all phenolic compounds, UV detection was performed at $225 \mathrm{~nm}$.

\section{Analysis of real samples}

Once the optimized MISPE experimental conditions had been established, surface water samples were used to demonstrate the applicability of the synthesized materials for the extraction of bisphenol A from real samples. In this way, surface water samples, from Odra River (Opole City, southern West Poland) were collected in $2.5 \mathrm{dm}^{3}$ amber glass bottles, filtered on $0.22-\mu \mathrm{m}$ cellulose acetate filters and stored at $4^{\circ} \mathrm{C}$ in the dark until measurement. The samples were fortified with the target analytes at concentration level $20 \mu \mathrm{g} / \mathrm{dm}^{3}$. Then, they were centrifuged for $10 \min \left(3000 \mathrm{rpm}, 20^{\circ} \mathrm{C}\right)$ to remove suspended matter. All the analyses, at least, have been carried out of triplicate. 


\section{Results and discussion}

\section{Influence of polymerization parameters on MIPs recognition abilities}

The proper selection of chemicals and stoichiometrical composition of polymerization mixture plays a crucial role in the imprinting process. It influences the molecular recognition properties of synthesized polymers. Solvent plays a critical role in the formation of the porous structure of molecularly imprinted polymers [51-53]. The polarity of solvents used in imprinting process affects the specificity of obtained polymers. Therefore, four molecularly imprinted polymers with methacrylic acid (MAA) as functional monomer and various porogenic solvents such as acetonitrile (MIP1), chloroform (MIP 2), toluene (MIP 3) and dichloromethane (MIP 4) were synthesized. To study the effect of the type of functional monomer, the other MIPs, both in acetonitrile, were synthesized, using 4-vinylpirydyne (MIP 5) and acrylamide (MIP 6). The obtained polymeric materials were then tested taking into account their recognition properties to extract BPA selectively from aqueous solutions.

In this case, the cartridges with proper sorbents (MIP or NIP) were first rinsed with $1 \mathrm{~cm}^{3}$ of water and $1 \mathrm{~cm}^{3}$ of methanol. Then aqueous spiked samples of BPA $\left(10 \mathrm{~cm}^{3}\right)$ at $0.5 \mu \mathrm{g} / \mathrm{cm}^{3}$ concentration level were passed through the MISPE cartridges at a flow rate of $1.5 \mathrm{~cm}^{3} / \mathrm{min}$. Under these conditions, bisphenol A was fully retained on all the MIPs and NIPs (Fig. 1).

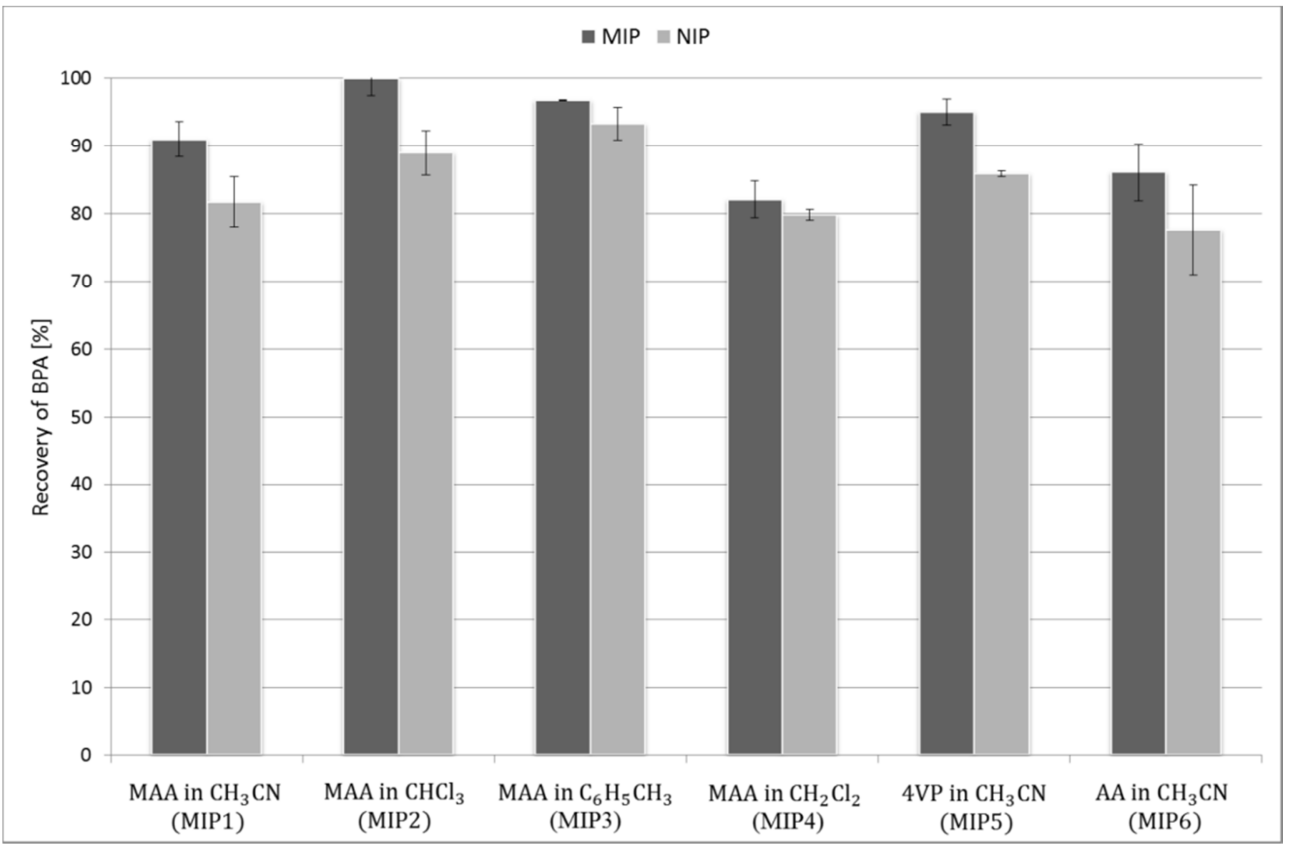

Fig. 1. Effect of porogenic solvent and type of functional monomer used in MIPs synthesis on bisphenol recovery A from aqueous samples. Sample: $0.5 \mu \mathrm{g} / \mathrm{cm}^{3}$ of bisphenol A, $10 \mathrm{~cm}^{3}$ volume. $(\mathrm{RSD}<5.4 \%, n=6)$ 
The obtained values of bisphenol A recovery were over $80 \%$. It has been found that an increase of dielectric constant of porogen solvent led to the slight improvement of binding properties of synthesized MIPs towards corresponding NIPs (polymeric materials with methacrylic acid as functional monomer named MIP 1, MIP 4, MIP 5 and MIP 6). This dependence is supposed to be related to the possibility of formation the strongest hydrogen bonds, established between the template and monomer with solvents at a higher dielectric constant. This effect could be stronger, especially in the case of MAA which capacity in forming hydrogen bond is greater than that of acrylamide (AA) or 4-vinylpirydyne (4VP) $[54,55]$. Nevertheless, the obtained selectivity of MIPs was not satisfied. Such small differences in values of BPA recovery between MIPs and NIPs (only 10-15\%), testified that the template molecules, during sorption process, were bind non-specifically with SPE sorbent, by hydrophobic interactions. In this case, BPA was bind not only in imprinting sites (cavities) but also on the surface of copolymers porous.

In the next step, the influence of the amount of applied cross-linker (EDMA) on MIPs recognition ability was investigated too. It has been observed that decreasing the concentration of crosslinker in polymerization mixture did not influence the quality of imprinted polymers (the size of particles) and its sorption and recognition properties advantageously. In this case, the type of porogenic solvent displayed to have a significant influence. When acetonitrile was applied as a porogen, the lowering of EDMA amounts from $20 \mathrm{mmol}$ to $10 \mathrm{mmol}$ caused that polymerization mixture reacted only partially. The obtained polymeric network (both MIP and NIP) were not dense enough and obtained sorbents were inappropriate for MISPE procedure. Their rigidity was not sufficient. Whereas an increase of cross-linker concentration (up to $30 \mathrm{mmol}$ ) did not improve a quality of the polymeric network, it is rigidity. Their application in MISPE procedure showed slight decreasing of BPA recovery (about 10-15\%). The opposite situation was observed when dichloromethane was used as porogen solvent. An increasing of EGMA concentration resulted in obtaining MIPs with a structure very much nailed together, making the polymer crumbling impossible, even at the EGMA concentration $20 \mathrm{mmol}$. The concentration of $10 \mathrm{mmol}$ of cross-linker allowed getting the satisfying polymeric material. Unfortunately, the desirable selectivity towards bisphenol A recovery from MIPs and adequate NIPs was still not satisfied.

An effect of template amounts on polymer properties also was tested. It was observed that increased of the molar ratio of template to EGMA decreased the recognition abilities of bisphenol A significantly by synthesized MIPs. The obtained recovery values were decreasing from $(92.2 \pm 0.9) \%$ to $(78.4 \pm 2.6) \%$, respectively for MIP 1 and MIP 10 . The lowering of template concentration from 4 to $2 \mathrm{mmol}$ did not influence the selectivity of applied MIP-SPE sorbents.

Finally, the temperature conditions of polymerization procedure and its influence on properties of obtained polymers as well as an application in MISPE extraction were studied, as temperature initiated the polymerization process. The free radical polymerization could run due to the presence of initiator molecules, a 1,1'-azobis-(cyclohexanecarbonitryle) $(\mathrm{ACHN})$. According to the literature data, its decomposition temperature is $37^{\circ} \mathrm{C}$ [56]. Therefore, an effect of temperature range from 40 to $80^{\circ} \mathrm{C}$ was studied. At a temperature of 40 or $50^{\circ} \mathrm{C}$, the MIP polymerization was observed, but the rigidity of obtained polymeric network was too strong, so it is crushing, and application for SPE experiments was impossible. Only temperature conditions above $60^{\circ} \mathrm{C}$ allowed obtaining molecularly imprinted polymers that could be used as sorbents in MISPE procedure. Further increasing 
of temperature up to $80^{\circ} \mathrm{C}$ did not result in rising of MIP properties towards bisphenol recognition and obtaining an effective MISPE sorption.

\section{MIP selectivity}

The most important parameter that characterized MIPs is their selective molecular recognition. Therefore, in further experiments, the potential of obtained imprinted polymers towards selective sorption of BPA from spiked aqueous samples was evaluated. Several alkylphenol compounds such as nonylphenol (4NP), 4-tert-octylphenol (t-OP) and 2-phenylphenol (2-PP) were selected to assess the selectivity of the MISPE protocol. In Figure 2, the obtained results of analyte recoveries on applied MIPs when loaded in water sample were presented.

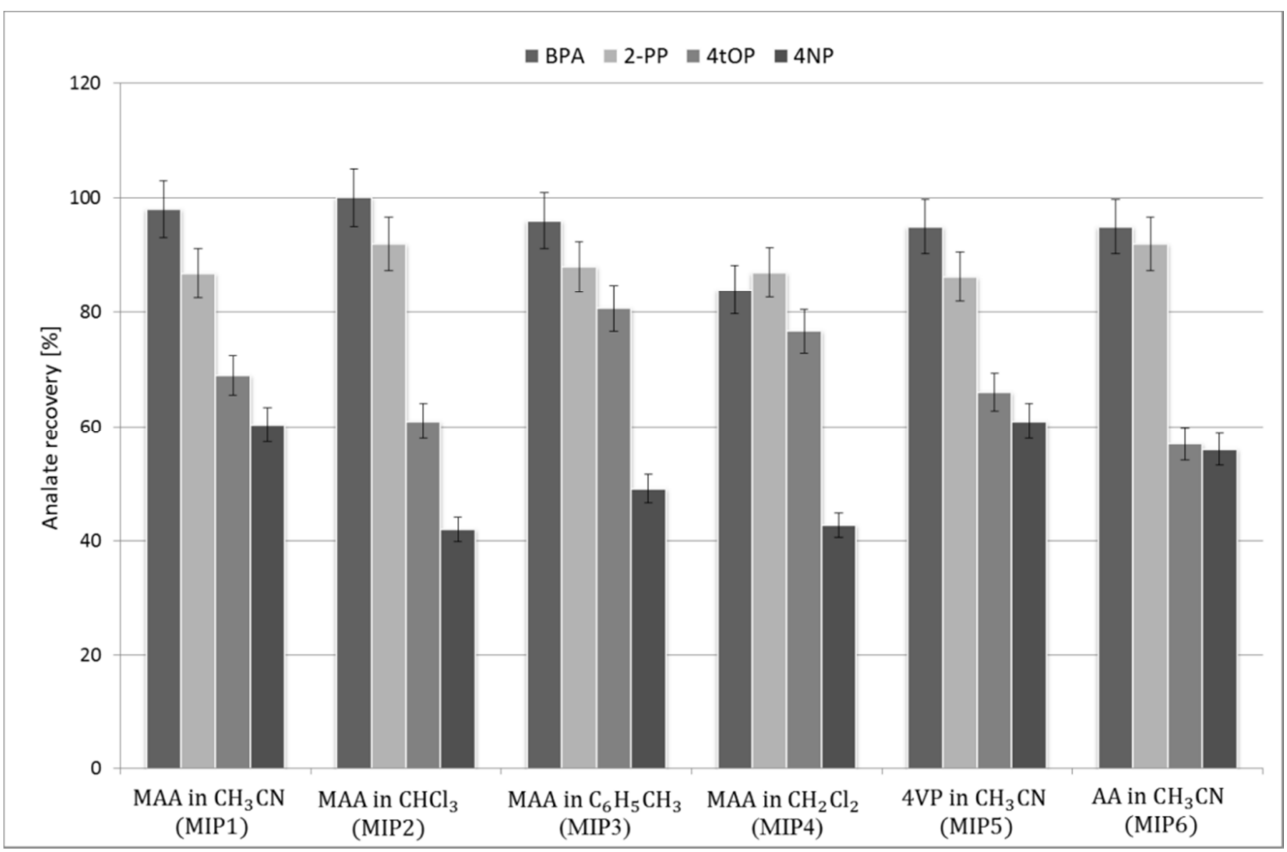

Fig. 2. Recovery values of bisphenol A and other alkylphenol compounds after running MISPE procedure. Sample: $10 \mathrm{~cm}^{3}$ volume of sample with analytes at concentration $0.5 \mu \mathrm{g} / \mathrm{cm}^{3}$ $(\mathrm{RSD}<4 \%, n=6)$

All the alkylphenols included the applied MIPs retained BPA (the highest value of recovery). However, the composition of study MIPs (a type of porogenic solvent and applied functional monomer) strongly influenced the binding properties of MISPE sorbents, considering the other analytes presented in the samples. Both BPA and 2-PP were extracted with the highest recovery in comparison to other aliphatic alkylphenols. The desirable selectivity, dedicated only for bisphenol A, was not observed. The similarity of chemical structure of 2-phenylphenol turned out to its effective recognition with imprinted cavities of used polymers. In the case of nonylphenol (NP) and 4-tert-octylphenol, the presence of long alkyl chain in its structure limited their effective interaction with recognition sites of 
applied molecularly imprinted polymers. The highest selectivity of the target analyte (BPA), at least toward 4-NP and t-OP, were observed in the case of four study polymers MIP 1, MIP 2, MIP 5 and MIP 6. The performed experiments also showed, that during the loading step no BPA and its analogs were detected in MISPE effluents, while in the case of breakthrough its presence in extraction with NISPE (non-imprinted polymers) was observed. This observation strongly indicated that the binding properties between MIPs and BPA in aqueous media were strong but nonspecific. Thus, further work was focused on the removal of non-specifically bonded analytes from MIPs sorbents resulted in decreasing their interaction with the selective cavities of molecularly imprinted polymers.

For this reason, the goal of the next experiments was to optimize the parameter of washing step of developed MISPE procedure with an application of different solvent types to remove the non-specifically bound alkylphenols from the polymers before elution step. The several solvents such as acetonitrile $\left(\mathrm{CH}_{3} \mathrm{CN}\right)$, dichloromethane $\left(\mathrm{CH}_{2} \mathrm{Cl}_{2}\right)$ and toluene $\left(\mathrm{C}_{6} \mathrm{H}_{5} \mathrm{CH}_{3}\right)$ were tested. Recoveries of bisphenol $\mathrm{A}$ and other alkylphenols during MISPE extraction with an application of various washing solvents were shown in Table 2 . At the beginning the optimization of washing step was performed on MIP 6, synthesized with acrylamide as functional monomer and $\mathrm{CH}_{3} \mathrm{CN}$ as a porogen, as a model polymeric material. The polymer was washed with successive aliquots of $0.5,0.75$ and $1.0 \mathrm{~cm}^{3}$ of the washing solvent. The obtained results indicated that $\mathrm{CH}_{3} \mathrm{CN}$ turned out to be not good washing solvents to attain selective extraction. Small differences appeared between BPA and other alkylphenols behavior when acetonitrile and dichloromethane were used as washing solvents. The significant losses of all analytes (including BPA) were observed with rising of washing volumes of $\mathrm{CH}_{3} \mathrm{CN}$. This behavior could be explained by the fact that acetonitrile may compete with analytes in hydrogen bonding formation with MIPs cavities. For comparison, an application of solvents with lower dielectric constant $\left(\mathrm{CH}_{2} \mathrm{Cl}_{2}\right.$ and toluene) during the washing step, the better binding properties of molecularly imprinted material were observed. Especially, a using of toluene allows an acceptable extraction of BPA with an efficient removal of the rest of analyzed alkylphenols.

Table 2

Recoveries of bisphenol A and other analytes in different washing solvents. MIP 6 synthesized with $\mathrm{AA}$ in $\mathrm{CH}_{3} \mathrm{CN}$ used as the model polymer. Analyte concentration was $0.5 \mu \mathrm{g} / \mathrm{cm}^{3}(\mathrm{RSD}<3.8 \%, n=6)$

\begin{tabular}{|c|c|c|c|c|}
\hline \multirow{2}{*}{ Washing solvent } & \multicolumn{3}{|c|}{ Recovery [\%] } \\
\cline { 2 - 4 } & BPA & 2-PP & t-OP & 4-NP \\
\hline Without washing & $95.0 \pm 1.7$ & $92.0 \pm 2.3$ & $57.0 \pm 2.8$ & $56.0 \pm 3.1$ \\
\hline $1.0 \mathrm{~cm}^{3} \mathrm{CH}_{3} \mathrm{CN}$ & $20.9 \pm 2.5$ & $29.7 \pm 3.8$ & $27.1 \pm 3.1$ & $19.5 \pm 2.6$ \\
\hline $0.75 \mathrm{~cm}^{3} \mathrm{CH}_{3} \mathrm{CN}$ & $32.4 \pm 9.6$ & $39.5 \pm 6.2$ & $40.4 \pm 6.8$ & $27.7 \pm 4.8$ \\
\hline $0.5 \mathrm{~cm}^{3} \mathrm{CH}_{3} \mathrm{CN}$ & $90.3 \pm 4.0$ & $91.7 \pm 6.5$ & $80.0 \pm 7.4$ & $51.3 \pm 5.8$ \\
\hline $0.75 \mathrm{~cm}^{3} \mathrm{CH}_{2} \mathrm{Cl}_{2}$ & $98 \pm 4.4$ & $60.2 \pm 5.9$ & $77.7 \pm 3.4$ & $74.1 \pm 2.7$ \\
\hline $0.5 \mathrm{~cm}^{3} \mathrm{CH}_{2} \mathrm{Cl}_{2}$ & $83.9 \pm 13.1$ & $80.1 \pm 11.2$ & $82.8 \pm 15.3$ & $79.2 \pm 13.6$ \\
\hline $1.0 \mathrm{~cm}^{3} \mathrm{C}_{6} \mathrm{H}_{5} \mathrm{CH}_{3}$ & $80.6 \pm 5.6$ & $2.4 \pm 1.8$ & $\mathrm{nd}$ & $\mathrm{nd}$ \\
\hline $0.75 \mathrm{~cm}^{3} \mathrm{C}_{6} \mathrm{H}_{5} \mathrm{CH}_{3}$ & $79.6 \pm 1.5$ & $7.3 \pm 1.2$ & $\mathrm{nd}$ & $1.20 \pm 0.08$ \\
\hline $0.5 \mathrm{~cm}^{3} \mathrm{C}_{6} \mathrm{H}_{5} \mathrm{CH}_{3}$ & $80.9 \pm 4.9$ & $8.9 \pm 3.4$ & $1.1 \pm 1.1$ & $2.40 \pm 0.09$ \\
\hline $0.5 \mathrm{~cm}^{3} \mathrm{C}_{6} \mathrm{H}_{5} \mathrm{CH}_{3}(\mathrm{NIP} 6)$ & $58.5 \pm 1.6$ & $29.6 \pm 1.7$ & $24.2 \pm 0.9$ & $20.9 \pm 0.5$ \\
\hline
\end{tabular}

The obtained results demonstrated that rising of $\mathrm{C}_{6} \mathrm{H}_{5} \mathrm{CH}_{3}$ volume during the washing step influences only the sorption of interferences compounds, whereas simultaneously the molecular imprinting effect was revealed and maximized. The toluene has turned out to be excellent washing solvent. It enabled to obtain good selectivity and high imprinting effect 
of BPA in comparison to other alkylphenols, t-OP, 4-NP, and 2-PP. During the washing procedure with $0.75 \mathrm{~cm}^{3}$ of $\mathrm{C}_{6} \mathrm{H}_{5} \mathrm{CH}_{3}$ and $1 \mathrm{~cm}^{3}$, the analytes such as t-OP and 4-NP was completely removed from the sorbents, whereas 2-phenylphenol, the most similar with its structure to bisphenol A, was extracted with very low effectiveness (recovery values below $10 \%)$.

The comparison of BPA preconcentration on MIP 6 and adequate NIP 6 demonstrated the selective sorption of BPA toward imprinted polymer. Two reasons of such situations could be. It might concern, both the different interaction between solvent, studied analytes and the surface of synthesized polymer as well as changes in the network structure of obtained molecularly imprinted sorbents treated with toluene. The differences of analytes recoveries, observed between MIP 6 and NIP 6, when the same MISPE procedure, including an application of washing step with $0.5 \mathrm{~cm}^{3}$ toluene, confirmed such phenomenon. Once, the non-imprinted sorbent was used, the ratio of BPA recovery towards other analytes was only 2:1. In the case of molecularly imprinted polymer (MIP6), the selectivity of bisphenol A sorption toward other phenolic compounds was 8:1. The obtained results indicated that treating the polymers (imprinted and non-imprinted) with various solvents could considerably modify the configuration of the polymeric network. The specific changes of structures in polymer sorbents are related to the porosity and flexibility of chains and molecular recognition of imprinted cavities. This phenomenon had been already described in the literature [57]. It concerned the experiments of solvent-induced structural changes that were observed in porous S-DVD copolymers. In these experiments, the samples of copolymers where well swollen in toluene placed into the small column and washed with for example methanol or water. In that studied, the porosity described by total pore volume $\left[\mathrm{cm}^{3} / \mathrm{g}\right]$ was changed for polymer pretreated with toluene and water, from 0.223 to 0.609 , respectively. The increase in surface area was observed. The changes in porosity were found to be reversible and reproducible.

a)

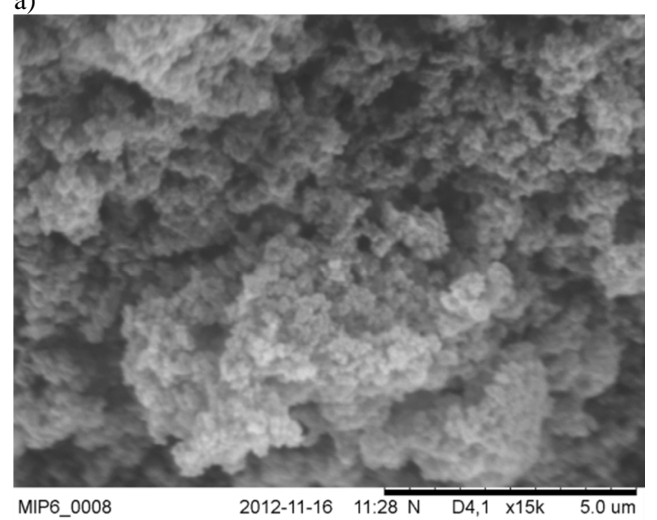

b)

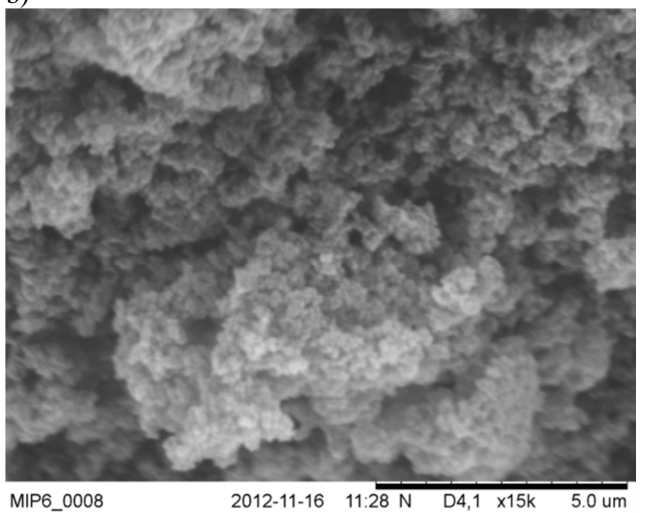

Fig. 3. The SEM image of BPA-imprinted polymer (MIP 6, prepared with AA in $\mathrm{CH}_{3} \mathrm{CN}$ ). a) MISPE without washing; b) MISPE with washing step with toluene. SEM image magnified 10,000-fold

In our studies, the morphological characteristic of the imprinted polymer (MIP 6) after an application of MISPE protocol (with or without an application of washing step with toluene) were evaluated by using scanning electron microscopy (SEM). The obtained 
scanning electron micrographs were presented in Figure 3. SEM analysis clearly showed the differences in surface morphology and internal structure of the sorbent (MIP 6) that was treated by toluene. Its surface was characterized by many smaller microporous and flow-through pores embedded in the network skeleton of MIP 6. In this case, the smaller size of microporous seemed to influence the binding ability of imprinted polymer significantly. The smaller microporous limited the access of other molecules to imprinted cavities; therefore the MIP 6 exhibited a specific recognition only for the template molecules.

Summing up, the volume of $0.75 \mathrm{~cm}^{3}$ of $\mathrm{C}_{6} \mathrm{H}_{5} \mathrm{CH}_{3}$ was chosen as the best washing step condition and applied for studies of sorption of BPA and other analytes on synthesized MIPs. An examination of elution degree for the MIP reveals, as expected, that recognition was higher for the template molecule (Fig. 4).

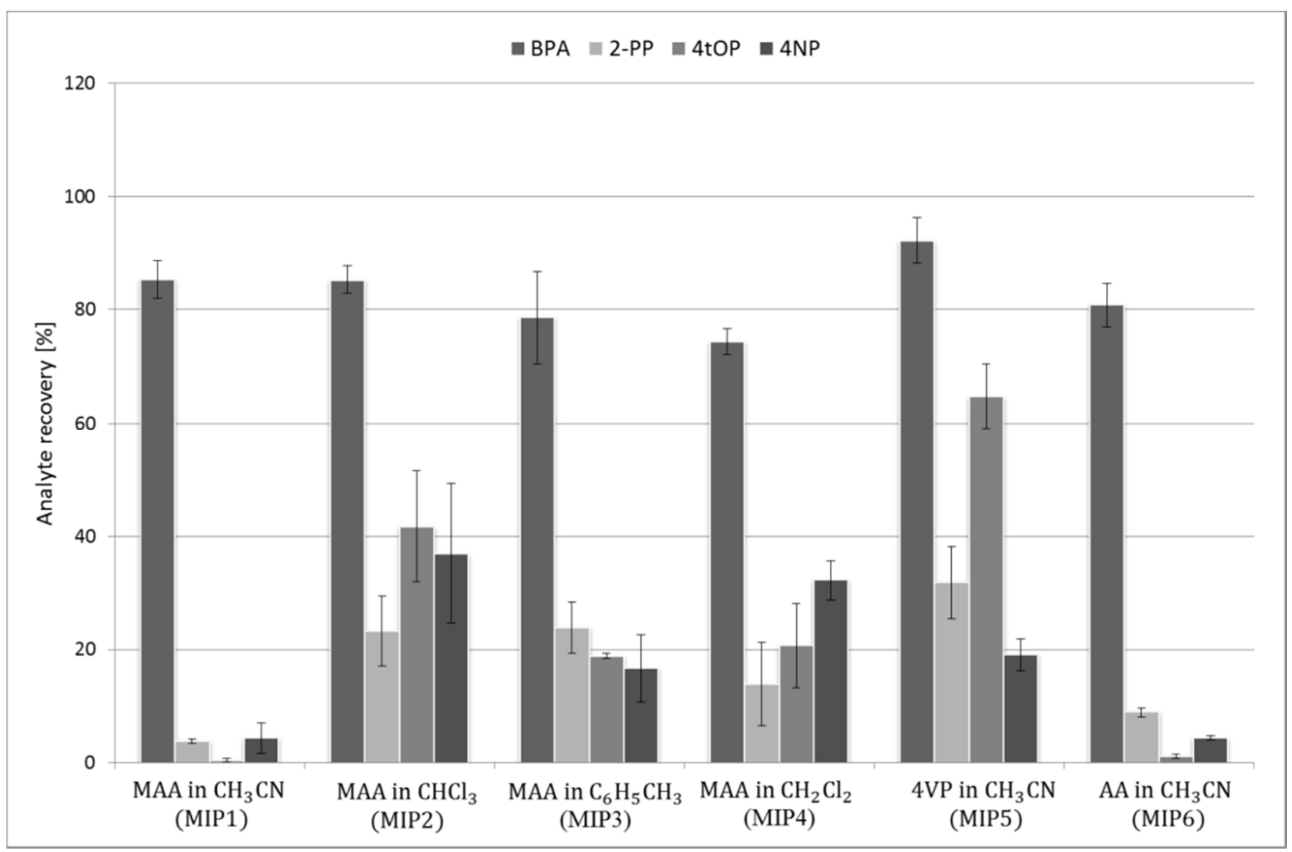

Fig. 4. Recoveries of bisphenol A and selected alkylphenols at optimized MISPE protocol using $0.75 \mathrm{~cm}^{3}$ of toluene during the washing step. Sample: $10 \mathrm{~cm}^{3}$ volume of sample with analytes at concentration $0.5 \mu \mathrm{g} / \mathrm{cm}^{3}$

The obtained results showed that the lowest amounts of interfering alkylphenols were retained on sorbents MIP 1 and MIP 6. In this case, t-OP was almost entirely stripped off in the washing step from both MIPs. In both cases, the BPA was extracted with high selectivity. These resulted confirm that obtained BPA-imprinted polymers prepared both in acetonitrile with MAA or AA as functional monomer could selectively separate and preconcentrate BPA from its analogs during optimized MISPE protocol. 


\section{Validation of analytical method}

To evaluate the applicability of the proposed MISPE procedures, the repeatability, linearity, and limits of detection (LOD) were investigated using HPLC-DAD as the detection system. For linearity studies, the calibration plots were prepared. For this case, 10 samples were spiked with BPA and APs to give final concentration 0.05, 0.1, 0.5, 1.0, 5.0 and $10 \mu \mathrm{g} / \mathrm{cm}^{3}$, and then extracted. The HPLC peak area counts were plotted against the respective analyte concentration to generate calibration curves. The calibration plots were linear over the range $0.05-10 \mu \mathrm{g} / \mathrm{cm}^{3}$ with correlation coefficient $\left(R^{2}\right) 0.9919-0.998$ for applied MISPE protocol. The limit of quantification (LOQ) and limit of detection (LOD) was defined as analyte signal-to-background noise $(\mathrm{S} / \mathrm{N})$ ratio. The LOD of the developed method without the application of washing step, for $10 \mathrm{~cm}^{3}$ sample volume, were found to be $25 \mu \mathrm{g} / \mathrm{dm}^{3}$ for BPA, and $50 \mu \mathrm{g} / \mathrm{dm}^{3}$ for 2-PP, $110 \mu \mathrm{g} / \mathrm{dm}^{3}$ for t-OP and $500 \mu \mathrm{g} / \mathrm{dm}^{3}$ for 4-NP. Whereas LOQ was respectively $70,150,330$ and $1513 \mu \mathrm{g} / \mathrm{dm}^{3}$ for BPA, 2-PP, t-OP, and 4-NP. In the case of MISPE procedure with an application of washing step with toluene, the LOD for $10 \mathrm{~cm}^{3}$ sample were found to be $25 \mu \mathrm{g} / \mathrm{dm}^{3}$ for BPA, and $140 \mu \mathrm{g} / \mathrm{dm}^{3}$. We found that an increase of the volume of extracted sample, from 10 up to $1000 \mathrm{~cm}^{3}$ resulted in improvement of LOD and LOQ (100 times fold) of studied analyte. These values were adequate for BPA and APs concentration level that was observed in the analyzed surface water samples (range $0.01-15 \mu \mathrm{g} / \mathrm{dm}^{3}$ ). The breakthrough volume of MISPE was greater than $1000 \mathrm{~cm}^{3}$ when $20 \mu \mathrm{g} / \mathrm{dm}^{3}$ mixed solutions were loaded. The repeatability of the analytical performance was studied for six replicate experiments of $10 \mathrm{~cm}^{3}$ of sample with $50 \mu \mathrm{g} / \mathrm{dm}^{3}$ BPA and APs standards solution with relative standard deviations (RSD) lower than 6\%.

\section{Analysis of real water samples}

Finally, the developed method was applied to the analysis of samples from Odra River (all originated from the city of Opole located at southwest of Poland). The extraction was performed with a use of polymer - MIP 6, synthesized with acrylamide as functional monomer and $\mathrm{CH}_{3} \mathrm{CN}$ as a porogenic solvent. The collected samples were spiked with appropriate amounts of study analytes (concentration $20 \mu \mathrm{g} / \mathrm{dm}^{3}$ ) and extracted via MISPE columns to examine the matrix effect. An aliquot of the collected samples was analyzed before spiking to determine possible background concentration of studied analytes (especially BPA). The study was carried out by performing six replicate experiments. The chromatograms of BPA determination in spiked surface water samples, including two variants of applied MISPE protocol, were presented in Figure 5. Once, the washing step (with toluene) was performed during the extraction procedure; only BPA could be selectively detected. Whereas all selected analytes, BPA, 2-phenylphenol, 4-NP, and t-OP were determined in analyzed samples if the MISPE procedure without any washing step was used. Those results clearly demonstrated that surface water matrices had no significant effect on the efficiency of developed method, which turned out to be suitable for analysis of trace level of BPA as well as APs from environmental samples.

The chromatogram of the analysis of unspiked and spiked surface water samples after optimized MISPE extraction procedure confirmed an effectivity of developed system toward trace analysis of BPA. The selectivity of applied procedure was strongly related to the application of washing step during the extraction process. The detected concentration of studied alkylphenols was $19 \mu \mathrm{g} / \mathrm{dm}^{3}$ for BPA and $71 \mu \mathrm{g} / \mathrm{dm}^{3}$ for 2-PP. The 4-NP and t-OP 
were not observed in studied surface water samples when developed MISPE procedure included washing step with toluene. Whereas, during MISPE extraction without an application of washing solvent, all selected APs were detected. The same MIP cartridges were able to be used for 20 consecutive cycles without any losses of molecular sorbent recognition abilities.

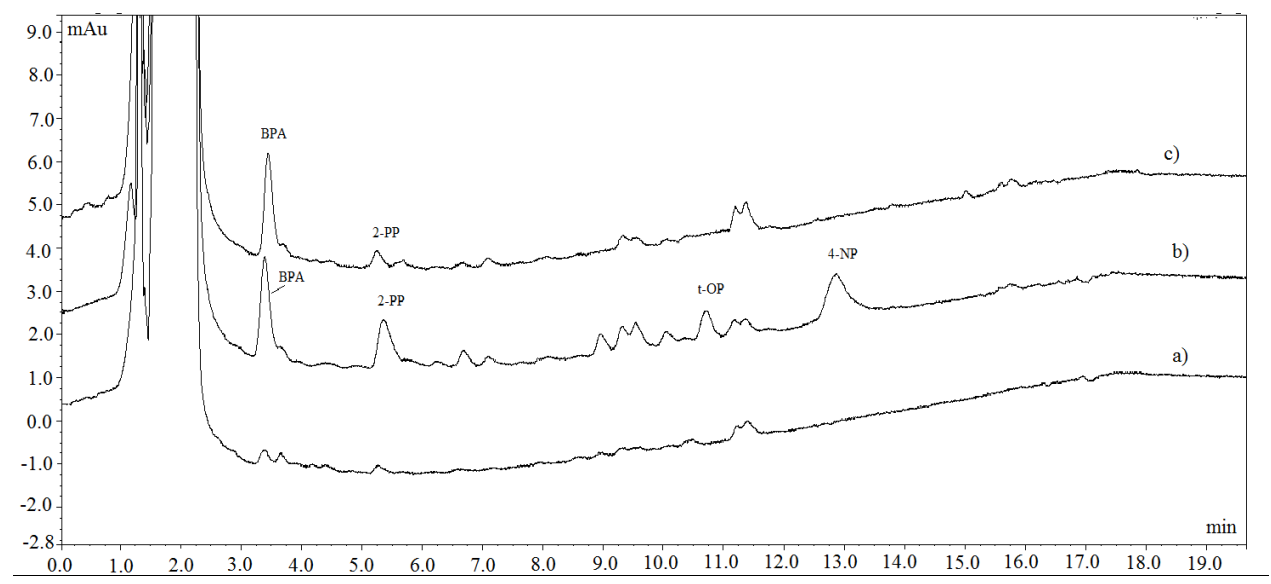

Fig. 5. Chromatograms obtained by on-column MIP-SPE for a $250 \mathrm{~cm}^{3}$ river water sample spiked with $20 \mu \mathrm{g} / \mathrm{dm}^{3}$ of BPA, 2-PP, 4-NP, and t-OP; a - unspiked water sample; b - spiked sample with MIP 6 without washing step; c - spiked sample with MIP6 with washing step using $0.5 \mathrm{~cm}^{3}$ of toluene

\section{Conclusions}

In this work, we described the synthesis and characterization of BPA-molecularly imprinted polymer used as a sorbent for solid phase extraction of bisphenol A. The developed MISPE method showed high selectivity towards target analytes if appropriate washing step was included during extraction. The developed washing and elution procedure enabled to obtain 50\% higher recovery of BPA from MIP with a comparison to NIP. The high selectivity and binding recognition of BPA (8:1) according to other structural analogs presented in the sample were observed. It was shown that acrylamide and methacrylic acid enhanced imprinted capacity when used as the functional monomer together with acetonitrile as porogenic solvent. The method has been successfully applied for the analysis of BPA in surface water samples showing its applicability for environmental analysis. The results indicated that depending on the requirements, the same molecularly imprinted sorbents might be used in two ways, once for selective extraction of BPA by using MISPE with washing step and another time for determination BPA together with other alkylphenols by using MISPE without any washing step. Good reproducibility for surface water samples with low matrix effect was obtained for both developed extraction procedure. Finally, the high selectivity provided by the synthesized MIP sorbent, made possible to develop a straightforward and low-cost LC-UV method whose performance in sensitivity and selectivity is similar or even better to that existing method based on the used molecularly imprinted polymers $[47,50]$. 


\section{References}

[1] Phillips KP, Tanphaichitr NJ. Toxicol Environ Health, B Crit Rev. 2008;11:188-220. DOI: 10.1080/10937400701873472.

[2] Soares A, Guieysse B, Jefferson B, Cartmell E, Lester JN. Environ Int. 2008;34:1033-1049. DOI: 10.1016/j.envint.2008.01.004.

[3] Scholz S, Klüver N. Sex Dev. 2009;3:136-151. DOI: 10.1159/000223078.

[4] Ge J, Cong J, Sun Y, Li G, Zhou Z, Qian C, Liu F. Bull Environ Contam Toxicol. 2010;84:401-405. DOI: 10.1007/s00128-010-9958-3.

[5] McLachlan JA. Andrology. 2016;4(4):684-94. DOI: 10.1111/andr.12206.

[6] Mearns AJ, Reish DJ, Oshida PS, Buchman M, Ginn T, Donnelly R. Water Environ Res. 2009;81:2070-2125. DOI: 10.2175/106143009X12445568400737.

[7] Postigo C, Kuster M, Villagrasa M, Rodríguez-Mozaz S, Brix R, la Farré M, et al. Liquid chromatography-mass spectrometry methods for analysis of endocrine-disrupting chemicals in wastewaters. In: Barceló D, Hansen P-D, editors. Biosensors for Environmental Monitoring of Aquatic Systems. Springer, Berlin/Heidelberg, Hdb Env Chem. 2009:5J:227-271. DOI: 10.1007/698_2009_18.

[8] Grün F, Blumberg B. Mol Cell Endocrinol. 2009;304:19-29. DOI: 10.1016/j.mce.2009.02.018.

[9] Ballesteros-Gómez A, Rubio S, Pérez-Bendito D. J Chromatogr A. 2009;1216:449-469. DOI: 10.1016/j.chroma.2008.06.037.

[10] Clouzot L, Marrot B, Doumenq P, Roche N. 17 $\alpha$-Ethinylestradiol: An endocrine disrupter of great concern. Analytical methods and removal processes applied to water purification. A review. Environ Prog. 2008;27;383-396. DOI: 10.1002/ep.10291.

[11] Di Lorenzo D, Rando G, Ciana P, Maggi A. Toxicol Sci. 2008;106:304-311. DOI: 10.1093/toxsci/kfn191.

[12] Hotchkiss AK, Rider CV, Blystone CR, Wilson VS, Hartig PC, Ankley GT, et al. Toxicol Sci. 2008;105:235-259. DOI: 10,1093/toxsci/kfn030.

[13] Mearns AJ, Reish DJ, Oshida PS, Ginn T, Rempel-Hester MA, Arthur C, et al. Water Environ Res. 2015;87(10):1718-816. DOI: 10.2175/106143015X14338845156380.

[14] McLachlan JA. Andrology. 2016;4(4):684-94. DOI: 10.1111/andr.12206.

[15] Al-Hiyasat AS, Darmani H, Elbetieha AM. Eur J Oral Sci. 2002;110:163-167. DOI: 10.1034/j.1600-0722.2002.11201.x.

[16] Sakaue M, Ohsako S, Ishimura R, Kurosawa S, Kurohmaru M, Hayashi Y, et al. Occup Health. 2001;43:185-190. DOI: 10.1539/joh.43.185.

[17] Krishnan AV, Stathis P, Permuth SF, Tokes L, Feldman D. Endocrinology (Philadelphia). 1993;132:2279-2286. DOI: 10.1210/endo.132.6.8504731.

[18] Sohoni P, Tyler CR, Hurd K, Caunter J, Hetheridge M, Williams T, et al. Environ Sci Technol. 2001;135:2917-2925. DOI: 10.1021/es000198n.

[19] Oehlmann J, Schulte-Oehlmann U, Tillmann M, Markert B. Ecotoxicology. 2000;9:383-397. DOI: 10.1023/a:1008972518019.

[20] Eramo S, Caldarella C, Lombardi V, Lombardo G, Sebastiani B. Dental Cadmos. 2011;79(6):357-64. DOI: 10.1016/j.cadmos.2011.01.006.

[21] Pulgar R, Olea-Serrano MF, Novillo-Fertrell A, Rivas A, Pazos P, Pedraza V, et al. Environ Health Persp. 2000;108(1):21-27. PMID: 10620520.

[22] Biles JE, McNeal TP, Begley TH. J Agri Food Chem. 1997;45:4697-4700. DOI: 10.1021/jf970518v.

[23] Choi IS, Cho JH, Park EJ, Park JW, Kim SH, Lee MG, et al. Neurosci Res. 2007;59;8-17. DOI: 10.1016/j.neures.2007.05.003.

[24] Mizuo K, Narita M, Miyagawa K, Suzuki T. Biomol Ther. 2010;18:125-134. DOI: 10.4062/biomolther.2010.18.2.125.

[25] Peng X, Wang Z, Mai B, Chen F, Chen S, Tan J, et al. Sci Total Environ. 2007;384:393-400. DOI: 10.1016/j.scitotenv.2007.05.043.

[26] Peng X, Yu Y, Tang C, Tan J, Huang Q, Wan Z. Sci Total Environ. 2008;397:158-166. DOI: 10.1016/j.scitotenv.2008.02.059.

[27] Gatidou G, Thomaidis NS, Stasinakis AS, Lekkas TD. J Chromatog A. 2007;1138:32-41. DOI: 10.1016/j.chroma.2006.10.037.

[28] Diao C, Yang X, Sun A, Liu R. Anal Methods. 2015;7(24):10170-6. DOI: 10.1039/c5ay02711j.

[29] Yu X, Xue J, Yao H, Wu Q, Venkatesan AK, Halden RU, et al. J Hazard Mater. 2015;299:733-9. DOI: 10.1016/j.jhazmat.2015.07.012.

[30] Ying GG, Kookana RS, Chen Z. J Environ Sci Heal B Pesticides. 2002;37:225-234. DOI: 10.5772/59885.

[31] Cao X-L, Perez-Locas C, Robichaud A, Clement G, Popovic S, Dufresne G, et al. Food Addit Contam Part A. 2015;32(12):2154-60. DOI: 10.1080/19440049.2015.1088663. 
[32] Kim WS, Do A, Yeh D, Cunningham J. Rev Anal Chem. 2014;33(1):59-77. DOI: 10.1515/revac-2013-0016.

[33] Yang Y, Lu L, Zhang J, Yang Y, Wu Y, Shao B. J Chromatogr A. 2014;1328:26-34. DOI: 10.1016/j.chroma.2013.12.074.

[34] Shao B, Han H, Tu X, Huang, L. J Chromatogr B. 2007;850:412-416. DOI: 10.1016/j.jchromb.2006.12.033.

[35] Liu J, Liang X, Jiang G, Cai Y, Zhou Q, Liu G. Talanta. 2003;60:1155-1161. DOI: 10.1016/S0039-9140(03)00216-9.

[36] Basheer C, Parthiban A, Jayaraman A, Lee HK, Valiyaveettil S. J Chromatogr A. 2005;1087:274-282. DOI: 10.1016/j.chroma.2005.03.014.

[37] Baggiani C, Baravalle P, Giovannoli C, Anfossi L, Giraudi G. Anal Bioanal Chem. 2010;397:815-822. DOI: 10.1007/s00216-010-3591-1.

[38] Yang Y, Yu J, Yin J, Shao B, Zhang J. J Agr Food Chem. 2014;62(46):11130-7. DOI: 10.1021/jf5037933.

[39] Ji Y, Yin J, Xu Z, Zhao C, Huang H, Zhang H, et al. Anal Bioanal Chem. 2009;395:1125-1133. DOI: 10.1007/s00216-009-3020-5.

[40] Jiang M, Shi Y, Zhang R, Shi Ch, Peng Y, Huang Z, et al. J Sep Sci. 2009;32:3265-3273. DOI: 10.1002/jssc.200900207.

[41] Kobayashi T, Takeda K, Ohashi A, Makoto M, Sugiyama S. Ther Apher Dial. 2009;13:19-26. DOI: 10.1111/j.1744-9987.2009.00651.x.

[42] Jiang X, Tian W, Zhao C, Zhang H, Liu M. Talanta. 2007;72:119-125. DOI: 10.1016/j.talanta.2006.10.006.

[43] Alexiadou DK, Maragou NC, Thomaidis NS, Theodoridis GA, Koupparis MA. J Sep Sci. 2008;31:2272-2282. DOI: 10.1002/jssc.200700643.

[44] Gu YY, Yu XJ, Peng JF, Chen SB, Zhong YY, Yin DQ, et al. J Chromatogr B. 2014;965:164-72. DOI: 10.1016/j.jchromb.2014.06.024.

[45] Kawaguchi M, Hayatsu Y, Nakata H, Ishii Y, Ito R, Saito K, et al. Anal Chim Acta. 2005;539:83-89. DOI: 10.1016/j.aca.2005.03.005.

[46] Navarro-Villoslada F, Vicente BS, Moreno-Bondi MC. Anal Chim Acta. 2004;504:149-62. DOI: 10.1016/s0003-2670(03)00766-9.

[47] Li J, Zhang X, Liu Y, Tong H, Xu Y, Liu S. Talanta. 2013;117:281-7. DOI: 10.1016/j.talanta.2013.09.022.

[48] Takeda K, Kobayashi T. Sci Technol Adv Mat. 2005;6:165-171. DOI: 10.1016/j.stam.2004.11.008.

[49] Herrero-Hernández E, Carabias-Martínez R, Rodríguez-Gonzalo E. Int J Mol Sci. 2011;12:3322-3339. DOI: 10.3390/ijms12053322.

[50] Mei S, Wu D, Jiang M, Lu B, Lim JM, Zhou YK, et al. Microchem J. 2011;98:150-155. DOI: 10.1016/j.microc.2011.01.003.

[51] Gruber HF. Prog Polym Sci. 1992;17(6):953-1044. DOI: 10.1016/0079-6700(92)90006-K.

[52] Joshi VP, Karmalkar RN, Kulkarni MG, Mashelkar RA. Ind Eng Chem Res. 1999;38:4417-23. DOI: 10.1021/ie990331o.

[53] Lasáková M, Jandera P. J Sep Sci. 2009;32:799-812. DOI: 10.1002/jssc.200800506.

[54] Cormack PAG, Elorza AZ. J Chromatogr B. 2004;804:173-182. DOI: 10.1016/j.jchromb.2004.02.013.

[55] Zheng N, Li YZ, Chang WB, Wang ZM, Li TJ. Anal Chim Acta. 2002;452:277-283. DOI: 10.1016/S0003-2670(01)01465-9.

[56] Mijangos I, Navarro-Villoslada F, Guerreiro A, Piletska E, Chianella I, Karim K, et al. Biosens Bioelectron. 2006;22:381-387. DOI: 10.1016/j.bios.2006.05.012.

[57] Galina H, Kolarz BN, Wieczorek P, Wojczyńska M. Brit Polym J. 1985;17:215-218. DOI: 10.1002/pi.4980170223. 